This item was submitted to Loughborough's Research Repository by the author.

Items in Figshare are protected by copyright, with all rights reserved, unless otherwise indicated.

\title{
Removal of micrometer size particles from surfaces using laser-induced thermocapillary flow: Experimental results
}

PLEASE CITE THE PUBLISHED VERSION

http://dx.doi.org/10.1016/j.jcis.2016.04.001

\section{PUBLISHER}

(c) Elsevier

\section{VERSION}

AM (Accepted Manuscript)

\section{PUBLISHER STATEMENT}

This work is made available according to the conditions of the Creative Commons Attribution-NonCommercialNoDerivatives 4.0 International (CC BY-NC-ND 4.0) licence. Full details of this licence are available at: https://creativecommons.org/licenses/by-nc-nd/4.0/

\section{LICENCE}

CC BY-NC-ND 4.0

\section{REPOSITORY RECORD}

Ivanova, Natalia A., Victor Starov, Anna Trybala, and V.M. Flyagin. 2016. "Removal of Micrometer Size Particles from Surfaces Using Laser-induced Thermocapillary Flow: Experimental Results". figshare. https://hdl.handle.net/2134/23210. 


\title{
Removal of micrometer size particles from surfaces using laser- induced thermocapillary flow: experimental results
}

\author{
Ivanova N.A. $^{1 *}$, Starov V.M. ${ }^{2}$, Trybala A. ${ }^{2}$, Flyagin V.M. ${ }^{1}$ \\ ${ }^{1}$ Photonics and Microfluidics Lab, Tyumen State University, Semakova 10, Russia, 625003 \\ ${ }^{2}$ Chemical Engineering Department, Loughborough University, LE11 3TU, UK \\ *Corresponding author: n.ivanova@utmn.ru
}

\section{Abstract \\ Hypothesis}

Reducing particle contaminations on solid and delicate surfaces is of great importance in a number of industries. A new non-destructive method is proposed, which is based on the laserinduced thermocapillary effect for the removal of micron size particles from surfaces. The cleaning mechanism is related to the surface-tension-driven flows produced by the laser heating of thin layer of a cleaning liquid deposited onto a surface contaminated with particles.

\section{Experiments}

Focusing the laser irradiation into the line laser beam allowed using this method for a large-scale cleaning of surfaces. Hexadecane was used as a cleaning liquid to remove micron-sized polyethylene, Teflon, talc and $\mathrm{Al}_{2} \mathrm{O}_{3}$ particles from surfaces of welding glass, carbolite and soft magnetic disc using the line beam of the IR laser.

\section{Findings}

A good cleaning efficiency was achieved for cases of polyethylene and Teflon particles on both the complete wettable welding glass and the low-wettable soft magnetic disc, while in case of oleophilic talc and $\mathrm{Al}_{2} \mathrm{O}_{3}$ particles the effectiveness of the cleaning method was lower on all three substrates investigated. The thermal influence of the laser irradiation on substrates used was measured with infrared camera. It was shown that temperature in the irradiated area during the long-time heating increases insignificantly and cannot cause any damage of the substrate. 
Keywords: laser cleaning, thermocapillary flow, particle removal

\section{Introduction}

Development of an efficient technology for removal of particles is of a considerable importance in various applications, including semiconductor manufacturing and IC industry, high-power optics, photonics, displays, data storage devices and other technologies dealing with critical surfaces [1, 2]. Among the variety of cleaning methods widely used in industry (chemical etching, ultrasonic and megasonic vibrations, high-pressure air/liquid jets, wiping, surfactants) [2-10], the laser-based cleaning techniques [4-11] have shown advantages for a number of applications owing to a flexibility, a contactless, a high precision and an ability to remove submicron-sized particles. The laser-cleaning methods, which rely on the pulsed laser heating of surfaces or particles, can be divided into two types: dry laser cleaning [4-7] and liquids assisted laser cleaning [4, 8-11]. In the case of dry laser cleaning particles are removed from the surfaces by rapid thermal expansion of solid substrates and/or particles that caused by the laser heating. In the liquid-assisted laser cleaning techniques particles are removed by the laser induced explosive vaporization of liquid film coating the substrate. However, despite some advantages of the latter two methods the high temperature developed by high power laser pulses could cause damage of surfaces and/or their functional layers by melting, mechanical cracking and other possible reasons. Even more than that, due to explosive thermal expansion of materials the laser-pulsed cleaning techniques results in an emission of cleaning products in the surrounding atmosphere that is critical for applications related to cleaning surfaces experiments inside a spacecraft.

Below a novel, soft and nondestructive method for removal of particles from solid surfaces is suggested. The method is based on the laser-induced thermocapillary phenomenon [12-16]. The mechanism of the thermocapillary phenomenon is as follows. If an incident CW- 
laser beam contacts a thin liquid layer deposited on the solid substrate then temperature of the substrate increases due to absorption of the laser irradiation. This results in a local increase in the temperature of the liquid and induces the temperature gradient along the liquid layer. This gradient creates a surface tension gradient along the free liquid interface, $\partial \gamma / \partial r=\gamma_{T}^{\prime}(\partial T / \partial r)$, which forces the liquid to flow away from the irradiated area (Fig. 1a), where $\gamma_{T}^{\prime}<0$ is the thermocapillary coefficient, which is negative for pure liquids, and $\partial T / \partial r$ is the temperature gradient. This flow is referred to as the laser-induced thermocapillary flow [12-16]. In the irradiated area the liquid layer becomes thinner and depending on the power of the laser beam the thermocapillary forces could cause a rupture of the layer [14-16] (Fig. 1b). As a result, the solid substrate becomes dry. Shifting the laser beam parallel to the substrate forces the liquid to recede in front of the moving laser beam. Thus, the shifting laser beam displaces the liquid along the surface. A focusing of the laser beam into the line beam enables a larger scale displacement of the liquid (Fig. 1c).

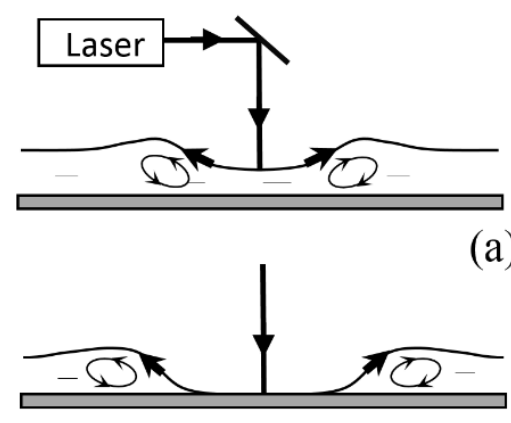

(b)

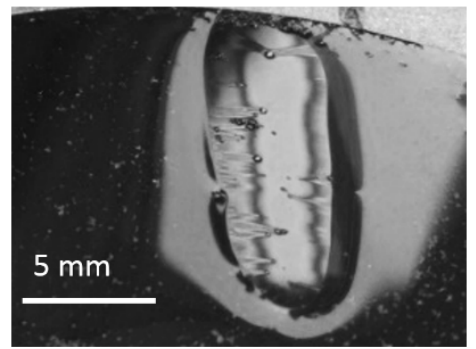

(c)

Fig. 1. (a) Schematic diagram of the laser-induced thermocapillary displacement of a liquid and (b) rupture of the liquid layer. (c) An image of the rupture of hexadecane layer on a magnetic disc under the irradiation by a line laser beam.

The particles to be removed originally are attached to the dry surface, after deposition of a liquid layer the particles becomes less connected to the solid surface and it is expected that the laser-induced thermocapillary flow can remove them from the solid surface and transfer with the 
flows. To validate applicability of the proposed approach the following experiments were carried out.

\section{Experimental section}

Particles of polyethylene (PE, average diameter 150 micron), Teflon (PTFE, average diameter $\sim 1$ micron) and talc (average diameter $\sim 10$ micron) were purchased from Sigma Aldrich (UK). Ceramic $\mathrm{Al}_{2} \mathrm{O}_{3}$ particles (average diameter $~ 120$ micron) were provided by our chemical department. The following solid substrates were used for cleaning, which capable of absorption of the laser irradiation: welding glass (optical density DIN12), carbolite and soft magnetic disc. The size of all substrates was approximately $3 \times 6 \mathrm{~cm}^{2}$. Low volatile hexadecane (Sigma Aldrich) was chosen as a cleaning liquid. The physical properties of hexadecane are the following [16]: the boiling temperature, $T_{b}=287^{\circ} \mathrm{C}$, the thermocapillary coefficient, $\gamma_{T}^{\prime}=$ $0.0842 \mathrm{mN} / \mathrm{m} \cdot \mathrm{K}$, the surface tension, $\gamma=27.64 \mathrm{mN} / \mathrm{m}$, and viscosity, $\mu=3.451 \mathrm{mPa} \cdot \mathrm{s}$ at $20^{\circ} \mathrm{C}$. Applying the sessile droplet method the wetting properties of substrates and particles in respect to hexadecane were investigated. The contact angles of hexadecane droplets $(<5 \mu l)$ were around $20^{\circ}$ on the magnetic disc, $<10^{\circ}$ on the carbolite, and complete wetting was observed on the welding glass. The wetting properties of PE and PTFE particles were measured using films of these polymers and the contact angles of hexadecane were found to be around 22 and $45^{\circ}$ on PE and PTFE films, respectively. The wettability of talc was estimated on surface of the dry talc powder compressed into a tablet. Hexadecane droplets soaked in the talc in a second after the deposition, which is caused by an imbibition into a porous tablet and oleophilic nature of talc. As far as $\mathrm{Al}_{2} \mathrm{O}_{3}$ powder is very granular, their wettability was roughly estimated by placing hexadecane droplet onto the surface of particles in a sample bottle. As in the case of talc, the droplet of hexadecane was immediately soaked up into the powder.

Particles were deposited onto the solid substrate by sieving using meshes with proper sizes of cell to reduce an aggregation of particles and achieve their uniform distribution over the 
solid substrates. The high-resolution images of substrates before and after cleaning were taken to estimate the efficiency of cleaning. The area under investigation was around $100 \mathrm{~mm}^{2}$.

The cleaning procedure was as follows. A hexadecane droplet $(\sim 0.1 \mathrm{ml})$ was deposited onto the area of the solid surface under investigation to completely cover all particles. A line beam of semiconductor laser (wavelength $1.5 \mu \mathrm{m}$, optical power $500 \mathrm{~mW}$ ) was directed perpendicularly to the liquid layer on the substrate. A length of projection of the line laser beam onto the substrate was around $1 \mathrm{~cm}$. Absorption of the laser irradiation lead to a heating of the liquid in the irradiated area, which resulted in the thermocapillary rupture of the layer and convective transfer of liquid from the heated to the colder area (Fig. 2a). Then the line laser beam was moved along the substrate inducing the thermocapillary displacement of the liquid over the substrate (Fig. 2b-c). The convective liquid flow moved the particles from the surface and transferred them along with the liquid flow. The displaced liquid was pumped out with a pipette after the liquid was removed from the investigated area of substrate (Fig. 2c-d). To achieve a maximum of the cleaning efficiency the cleaning procedure was repeated at least 5 times and the whole procedure took around 15 minutes. After completing the cleaning the investigated area of substrate was irradiated for 30 minutes to remove a residual very thin liquid film from the solid substrate. Using the images of substrate before and after the cleaning procedure the area of substrate covered by particles was estimated by counting of the pixels. The cleaning efficiency was estimated using the following equation:

$$
K=\frac{S_{0}-S}{S_{0}} \cdot 100 \%
$$

where $S_{0}$ is the area of the substrate covered by particles before cleaning, $S$ is the area of substrate covered by particles after cleaning. 

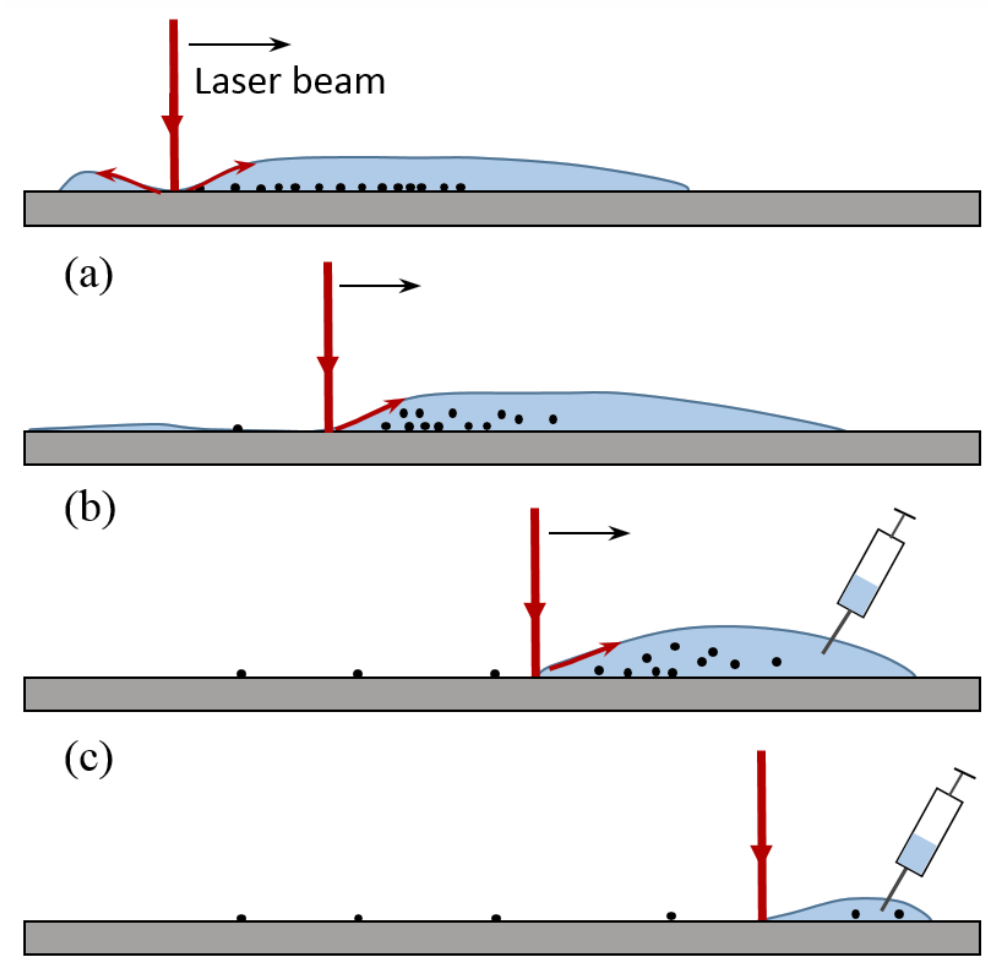

(d)

Fig. 2. Schematic diagram of steps of the cleaning procedure: (a) the thermocapillary rupture of the layer; (b-d) the thermocapillary displacement of the liquid with particles caused by the motion of the laser beam along the substrate; (c-d) pumping out the cleaning liquid.

For the estimation of the thermal influence of laser irradiation on substrate, the temporal and spatial distributions of surface temperature was investigated using infrared camera (FLIR A655sc, spectral range $7.5-14 \mu \mathrm{m}$, accuracy $\pm 2{ }^{\circ} \mathrm{C}$ of reading). The time evolution of surface temperature of substrate was measured starting from the switch on the laser until temperature in the center of the laser beam projection on a surface has reached a constant magnitude. After that the laser was switched off and the relaxation of surface temperature of substrate was monitored until it decreased to the room temperature. Fig. S1 shows the growth of surface temperature of welding glass (a), carbolite (b) and soft magnetic disc (c) during the heating with the laser beam. Fig. S2 shows the relaxation of the surface temperature for those substrates. 


\section{Results and discussion}

\subsection{Experimental results}

Fig. 3 illustrates the results of application of the laser-induced thermocapillary effect for cleaning of surfaces from the particulates: (a) talc particles on a welding glass, (b) PE particles on a soft magnetic disc. In Fig. 4 the cleaning efficiency of the laser-induced thermocapillary effect for all types of substrates and particles is shown. Fig. 4 shows that the proposed method is more effective in the case of PTFE and PE particles on welding glass and magnetic disc. In the case of talc particles on all substrates, the cleaning efficiency is low and ranges in between $30-45 \%$. Interesting results were obtained for $\mathrm{Al}_{2} \mathrm{O}_{3}$ particles: a satisfactory cleaning efficiency around 30 and $60 \%$ found on the carbolite and the magnetics disc, respectively, but on the welding glass, the cleaning efficiency was almost zero. In the course of the cleaning experiments, the following motions of particles in the layer and near the border of rupture were observed. (i) The sliding and/or rolling motion of all particles within the liquid layer along the substrate. (ii) The transport of particles with the receding/advancing contact line in zone of the thermocapillary rupture for the oleophobic particles. Note that big clusters of particles do not move and remain to adherent to the surface.

\subsection{The cleaning mechanism}

In general, there are three types of adhesion forces (van der Waals, electrostatic and capillary pressure forces), which attach small particles to surfaces. However, an immersion of particles in oil medium makes electrostatic forces to become negligible.

The cleaning forces in the liquid-assisted cleaning process could be the following: the hydrodynamic forces caused by the liquid flow around the particles $[17,18]$ and the capillary forces caused by the advancing/receding contact angles of the liquid layer [11]. The liquid flow in our case is the laser-induced by thermocapillary flows. Since the hydrodynamic forces depend on the flow velocity, then the higher flow velocity the better the cleaning efficiency. In the case 
under consideration the flow velocity is determined by the thermal surface tension gradient, $d \gamma / d r=\gamma_{T}^{\prime} d T / d r$, where $d T / d r$ is the temperature gradient caused by the laser beam on the hexadecane layer. To estimate the surface tension gradient the temperature gradients were calculated from IR measurements (Fig. S1) as slopes of the curves of temperature versus distance, which correspond to the location of the laser beam. The estimation of the $d \gamma / d r$ yields the following values: $0.8 \mathrm{~N} / \mathrm{m}^{2}$ on the soft magnetic disc, $0.34 \mathrm{~N} / \mathrm{m}^{2}$ on the carbolite and 0.2 $\mathrm{N} / \mathrm{m}^{2}$ on the glass. These values mean that the cleaning process has to be more efficient on the soft magnetic disc. Indeed Fig. 4 shows that on the magnetic disc the overall cleaning efficiency is higher for all particles in comparison with the other substrates. For this reason, the good cleaning can be expected on the carbolite surface; however, as it seen from Fig. 4, for PTFE and PE particles the cleaning efficiency is lower than that on the welding glass. The latter could be associated with the large-scale roughness and presence of pinholes in a top layer of the carbolite substrate that could lead to entrapment of particles and prevent their removal. The other reason for the good removal of PTFE and PE particles from the welding glass is the contribution of an extra cleaning capillary force at the leading edge on laser beam (Fig. 2). Indeed, according to Leenaars [11] the capillary force, $F_{c}$, generated by the moving interfacial liquid-solid boundary is equal to:

$$
F_{c}=\pi D \gamma \sin ^{2}\left(\frac{\theta}{2}\right) \cos \alpha
$$

where $\gamma$ is the surface tension of the liquid, $\theta$ is the contact angle between the liquid and the particle, and $\alpha$ is the contact angle of the liquid on the substrate. In our case, this kind of force works at the receding three-phase contact line (Fig. 2). According to Eqn. (2) the cleaning procedure is more efficient in case the highly wettable substrate and low wettable particles. Analysis of the wetting properties of particles and surfaces given in the experimental section shows that the cleaning capillary force can significant contribute to the removal of moderately oleophobic particles such as PTFE and PE from the oleophilic welding glass. The latter is in an 
agreement with our experimental results (Fig. 4): high cleaning efficiency was found for these particles on the glass surface.

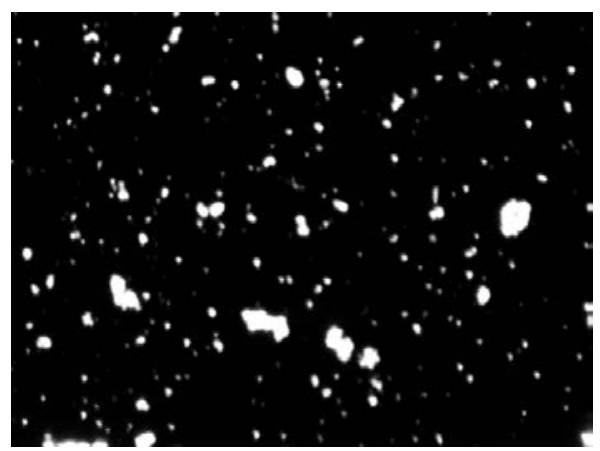

Before cleaning

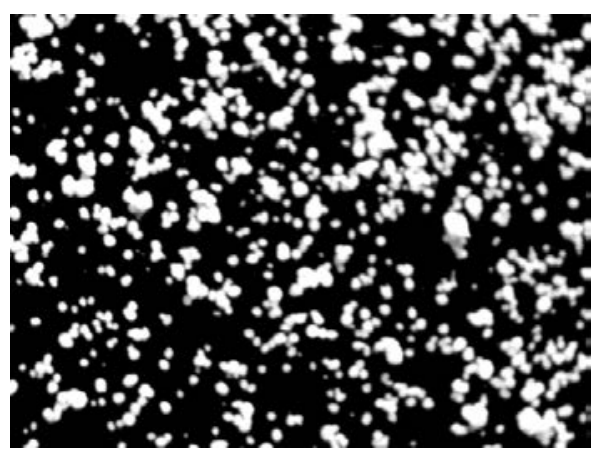

Before cleaning

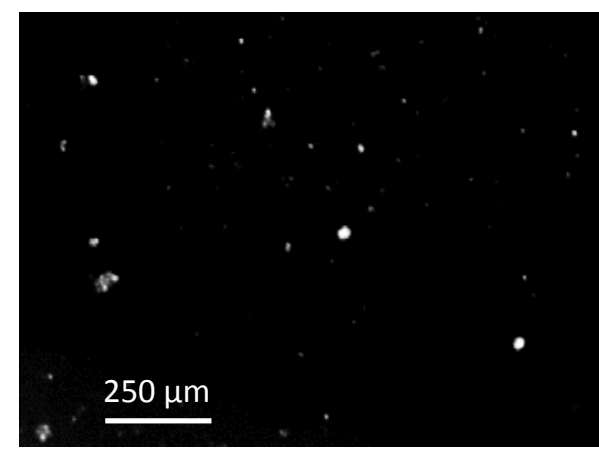

After cleaning

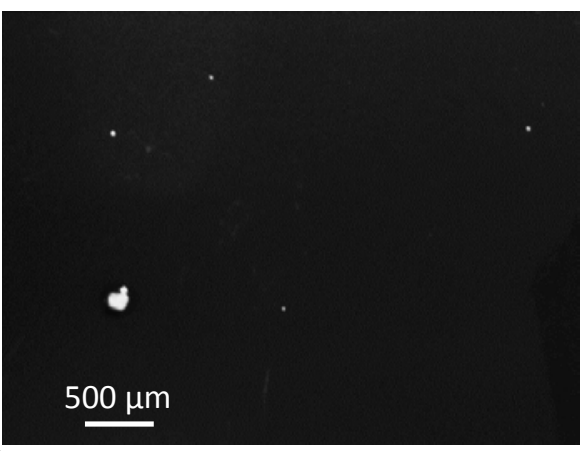

After cleaning

Fig. 3. Images of substrates before and after cleaning by the laser-induced thermocapillary method. (a) Talc particles on a welding glass. (b) PE particles on a soft magnetic disc.

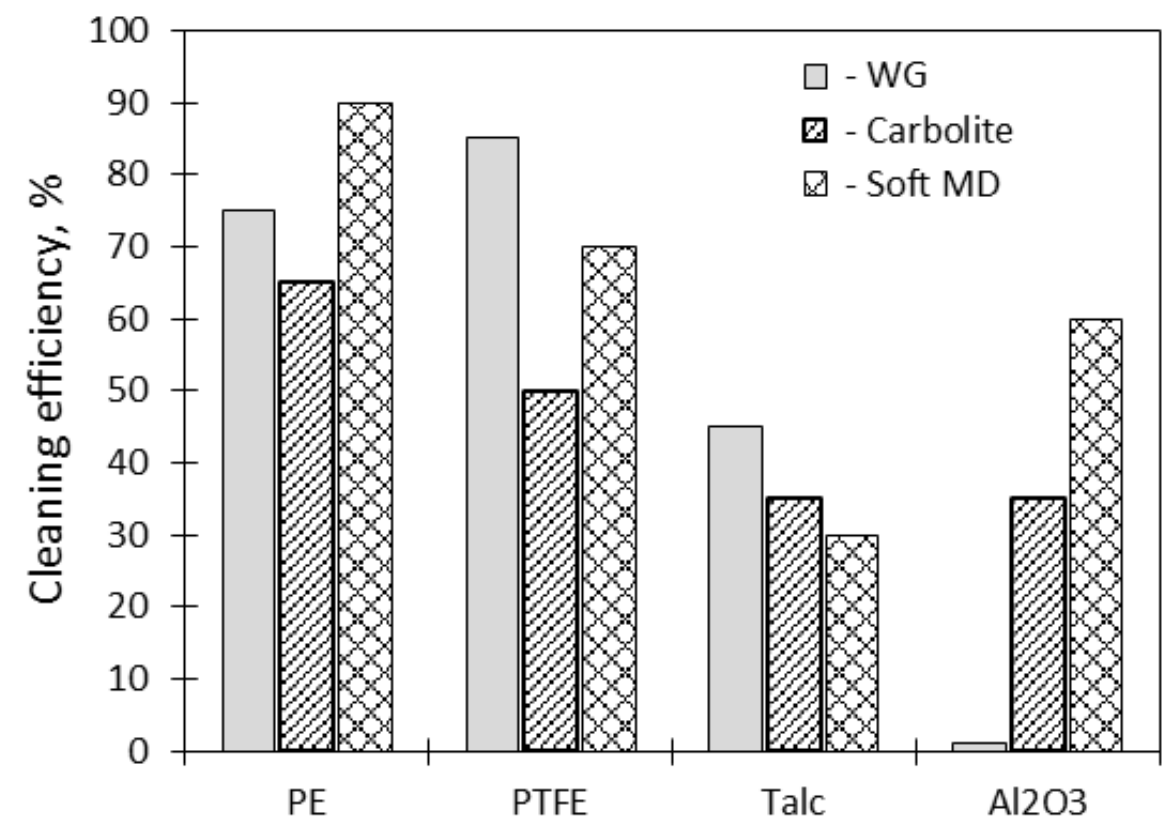

Fig. 4. Efficiency of removal of PE, PTFE, talc and $\mathrm{Al}_{2} \mathrm{O}_{3}$ particles from welding glass (WG), carbolite and soft magnetic disc (MD) by the laser induced thermocapillary flow. 


\subsection{Analysis of the thermal effect}

As it mentioned above the thermal impact on the substrate caused by the laser beam is a crucial parameter because of a danger of destruction of the surface. In Fig. 5 the dependencies of temperature of substrates in the center of projection of the line laser beam are presented. In the case of the welding glass and the carbolite, temperature reached maximum values close to $45^{\circ} \mathrm{C}$ and $60^{\circ} \mathrm{C}$, respectively. The heating rate is slow and takes several minutes after the beginning of the irradiation to reach the maximum value. However, on the soft magnetic disc temperature increased much faster (in ten of seconds) and reached a maximum value around $80^{\circ} \mathrm{C}$. The difference in the maximum temperatures is caused by the different ability of those materials to absorb the laser irradiation. The temperature curves in Fig. S1 show that on the welding glass and the carbolite the strong lateral dissolution of heat takes place, but no heat dissolution occurs on the magnetic disc. This is associated with the dissipation of heat laterally and in depth of substrate (the thickness of both the welding glass and carbolite is $3 \mathrm{~mm}$ ) caused by the thermal conductivity of material. The magnetic disc (60 microns thick) is a polymeric material having a low thermal conductivity that prevents the heat dissipation and allows the high temperature gradient in comparison with two other substrates. It is important to note, that the magnitude of the temperature gradient has a directly influence on the hydrodynamic cleaning force. Thus, the thermal properties of substrates and their thicknesses affect the cleaning efficiency of the proposed method. Nevertheless, it has to be noted that the laser-induced thermocapillary method enables the removal of micron-size particles at very low temperatures in contrast to the traditional laser-pulsed cleaning methods $[8,10]$ and cannot cause any destruction of the surfaces. 


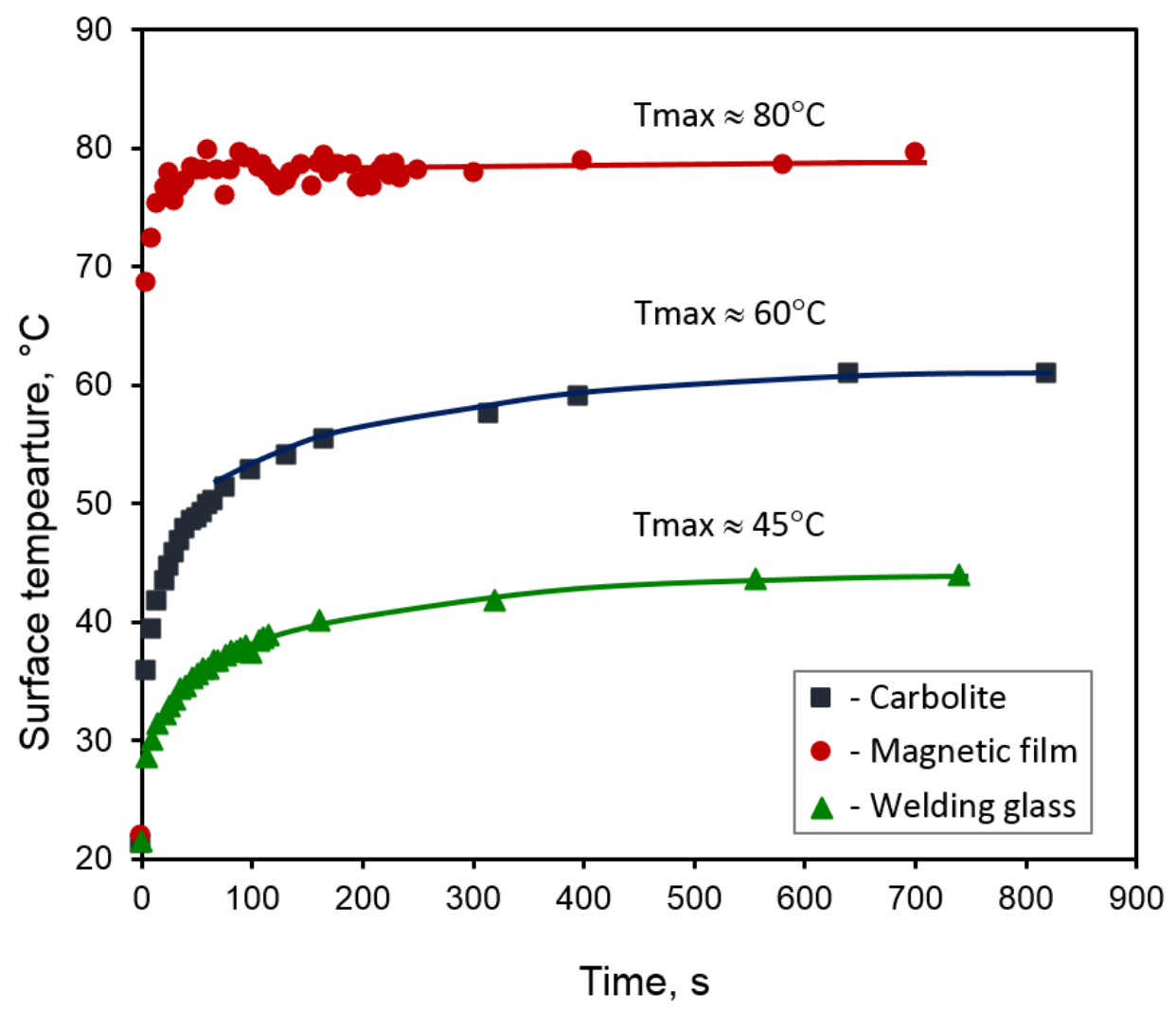

Fig. 5. Time evolution of the surface temperature in the center of projection of laser beam on the solid substrates $(\bullet-$ soft magnetic disc, $\boldsymbol{\square}$ - carbolite and $\boldsymbol{\Delta}$ - welding glass).

\section{Conclusions}

The soft and non-destructive laser-based cleaning method has been proposed to remove the micron-size particles from solid surfaces without causing thermal damage to these surfaces. The method is based on using the thermocapillary flows [12-16] induced by the line laser beam heating of the thin liquid layer deposited on the surfaces to be cleaned. The mechanism behind the laser-induced thermocapillary cleaning is relied on the hydrodynamic forces and the capillary force [11] acting on particles. The hydrodynamic forces are generated by the thermocapillary flows of the liquid and responsible for removal of particles within the liquid layer. The capillary force arise at the advancing edge of the laser beam and then acts on low-wettable particles at the moving three-phase contact line. It was shown that for the cases when both the hydrodynamic and capillary forces acting on particles (for example, low-wettable PE and PTFE) the proposed 
cleaning method is more efficient. The experimental results obtained are in good agreement with this force analysis. Measurements of the thermal influence of the laser irradiation onto the surfaces have clearly demonstrated that the laser-induced thermocapillary cleaning method operates at temperatures that are one order of magnitude lower in comparison with those of the laser-pulsed cleaning methods $[8,10]$ and do not cause any damage of surfaces.

The cleaning efficiency of the proposed method is affected by wetting propeties of both particles and substrates, as well as the value of surface tension gradient induced by the laser heating: the proper combination of these parameters enables not only to improve the cleaning effectivness of the laser-induced thermocapillary method, but also will open a possibility to extend its applicability to submicron particles and nanoparticles. The thermocapillary flow induced by the line laser beam can be considered as a very promising tool enabling the largescale cleaning of solid substrates at low cleaning temperatures, which can be employed in various applications, where delicate, hardly accessible surfaces have to be cleaned from particles.

\section{Acknowledgements}

This research was supported by the Royal Society UK - the RFBR (Grant no. 14-01-92602 KO_a) Russia collaboration grant, European Space Agency MAP EVAPORATION project, CoWet EU project and COST MP1106 EU project. N. Ivanova was also supported by the Ministry of Education and Science of the Russian Federation (Grant no. 1019).

\section{Appendix A. Supplementary material}

Supplementary data associated with this article can be found, in the online version, at ...

\section{References}


[1] Developments in surface contamination and cleaning. Vol. 2. Elsevier 2010. Eds.: Kohli R., Mittal K.L.

[2]. Particle adhesion and removal. Eds.: K.L. Mittal, R. Jaiswal. Scrivener Publishing, Wiley (New Jersey) 2015.

[3] F.M. Mahdi, T.E. Record, C.A. Amadi, F.O. Emmanuel, N.A. Ivanova, A. Trybala, V.M. Starov, Colloid and Interface Science Communications, 6 (2015) 13-16.

[4] A.C. Tam, W.P. Leung, W. Zapka, W. Ziemlich, J. Appl. Phys. 71 (1992) 3515 - 3523.

[5] Y.W. Zheng, B.S. Luk’yanchuk, Y.F. Lu, W.D. Song, Z.H. Mai, J. Applied Phys. 90 (2001) $2135-2142$.

[6] C. Curran, J.M. Lee, K.G. Watkins, Optics and Lasers in Engineering 38 (2002) 405 - 415.

[7] W.D. Song, M.H. Hong, H.L. Koh, W.G. Wang, Y.W. Zheng, Y.F. Lu, T.C. Chong, Appl. Surface Sci. 186 (2002) 69-74.

[8] Y.F. Lu, Y. Zhang, Y.H. Wan, W.D. Song, Appl. Surface Sci. 138-139 (1999) 140 - 144.

[9] Daehwan Ahn, Jeonghong Ha, Dongsik Kim, Appl Surface Sci. 265 (2013) 630 - 636.

[10] M. She, D.S. Kim, C.P. Grigoropoulos, J. Appl. Phys. 86(11) (1999) 6519-6524.

[11] A.F.M. Leenaars, Methods of Removing Undesired Particles from a Surface of a Substrate, US Patent (1988) 4,781,764.

[12] G. Da Costa, Appl. Optics 32 (1993) 2143 - 2151.

[13] S.P. Karlov, D.A. Kazenin, B.I. Myznikova, I.I. Wertgeim, J. Non-Equilib. Thermodyn. 30 (2005) $283-344$.

[14] H.M.J.M. Wedershoven, C.W.J. Berendsen, J.C.H. Zeegers, A.A. Darhuber, Appl. Phys. Lett. 104 (2014) 054101.

[15] B.A. Bezuglyi, N.A. Ivanova, A.Yu. Zueva. J. Appl. Mech. Tech. Phys. 42(3) (2001) 493496.

[16] B.A. Bezuglyi, O.A. Tarasov, Opt. Spectrosc. 94(2) (2003) 230 - 234.

[17] G.M. Burdick, N.S. Berman, S.P. Beaudoin, Thin Solid Films. 488 (2005) 116-123. 
[18] H-C. Wang, Aerosol Science and Technology. 13 (1990) 386-393. 


\section{Figure captions}

Fig. 1. (a-b) Schematic view of the laser-induced thermocapillary displacement of a liquid and the rupture of the liquid layer. (c) An image of the rupture of hexadecane layer on a magnetic disc under the irradiation with the laser line.

Fig. 2. Schematic view of the steps of the cleaning procedure: (a) the thermocapillary rupture of the layer; (b-d) the motion of the laser beam along the substrate causing the thermocapillary displacement of the liquid with the particles; (c-d) the pumping out the cleaning liquid.

Fig. 3. Images of substrates before and after cleaning by the laser-induced thermocapillary method. (a) Talc particles on a welding glass. (b) PE particles on a soft magnetic disc.

Fig. 4. Efficiency of removal of PE, PTFE, Talc and $\mathrm{Al}_{2} \mathrm{O}_{3}$ particles from welding glass (WG), carbolite and soft magnetic disc (MD) by the laser induced thermocapillary flow.

Fig. 5. Time evolution of the surface temperature in the center of projection of laser beam on the solid substrates ( $\bullet$ - soft magnetic disc, $\boldsymbol{\square}$ - carbolite and $\boldsymbol{\Delta}$ - welding glass).

Fig. S1. The growth of surface temperature of welding glass (a), carbolite (b) and soft magnetic disc (c) during the heating with the laser beam.

Fig. S2. The relaxation of the surface temperature for (a) welding glass, (b) carbolite and (c) soft magnetic disc. 